\title{
Induction of fibroblasts to neurons through adenoviral gene delivery
}

Cell Research (2012) 22:436-440. doi: 10.1038/cr.2011.185; published online 22 November 2011

\section{Dear Editor,}

The direct conversion of mouse fibroblasts to neurons [1] was developed while many scientists were interested in neuronal differentiation using induced pluripotent stem cells (iPSCs) [2]. This direct reprogramming skips a pluripotent state, making patient- and/or disease-specific cell therapy much faster and more feasible. Recent reports have indicated that mouse or human fibroblasts could be directly converted to a number of different cell types, such as cardiomyocytes, blood progenitor cells, hepatocyte-like cells, neural progenitors and specific dopaminergic neurons [3-9]. However, all of the studies in this field have employed the retroviral or lentiviral vector delivery system. These genome-integration viruses may cause insert mutations and activation of tumor-related genes; therefore, it is necessary to generate neurons with a non-integrating system. Concerning transcription factors, the reported combination of three neural-specific transcription factors, Ascll, Brn2 and Mytll (ABM), or the three combined with NeuroD1, can convert mouse and human fibroblasts to functional neurons $[1,4,5]$. It has also been reported that Ngn2 alone could direct astrocytes into neurons $[10,11]$. Therefore, it is possible that a different combination of transcription factors could induce fibroblasts to neurons as well. In this study, we used non-integrating adenoviruses carrying a different combination of transcription factors of Ascll, Brn2 and Ngn2 (ABN) for the direct conversion of mouse embryonic and adult fibroblasts to functional neurons.

The mouse cDNAs of Ascl1, Brn2, Ngn2 and Myt1l were cloned into a modified commercial adenoviral vector (Invitrogen, pAd/CMV/V5-DEST ${ }^{\mathrm{TM}}$ ) under the control of the CMV promoter (Supplementary information, Figure S1 and Data S1). The adenoviral backbone vector carried an enhanced green fluorescent protein (eGFP) reporter gene, which facilitated the calculation of virus titers and the tracing of induced neuronal (iN) cells during the entire course of transdifferentiation. The mouse embryonic fibroblasts (MEFs) were isolated with great care to exclude neural tissue as previously described [1]
(Supplementary information, Data S1). It was found that the uninfected MEFs were fibronectin positive (Supplementary information, Figure S2A). No pre-existing neurons, astrocytes or neural progenitor cells were detected, as demonstrated by immunocytochemistry with specific markers for Tuj1, GFAP, and Nestin, respectively (Supplementary information, Figure S2B-S2D). However, in the uninfected MEFs, we observed a few Tuj1-positive cells (5-12 out of 100000 cells) that morphologically appeared to be fibroblasts (Supplementary information, Figure S2B). MEFs were infected once daily for 2 days consecutively with three groups of adenoviruses: ABM, $\mathrm{ABN}$ and control eGFP (ctrl-eGFP) (Figure 1A).

The GFP expression level helped us observe changes of MEFs during the whole process of transdifferentiation. At 1 day post infection (dpi), the infection efficiency was estimated to be approximately $80 \%-90 \%$ by fluorescence, and the MEFs infected displayed typical fibroblast morphology (Supplementary information, Figure S3A). At 3-5 dpi, the infected MEFs began to show the morphological appearance of neurons with short and slender projections (Supplementary information, Figure S3B). At 7-10 dpi, more cells with complex processes were observed (Supplementary information, Figure S3C). At 13$18 \mathrm{dpi}$, neuronal-like cells with long processes forming a complex network with each other were readily observed (Supplementary information, Figure S3D).

Immunostaining with the neuronal marker Tuj1 was performed at 13-15 dpi. iN cells were defined as Tuj1positive cells with a neuronal morphology, including round cell bodies and an extension of at least one thin process three times longer than their cell bodies. This experiment demonstrated that the ABN combination of transcription factors could generate iN cells, which appeared as typical neurons in morphology with long, complex processes (Figure 1B and 1C, Supplementary information, Figure S4A). The previously reported transcription factors of ABM combination could also reprogram MEFs to iN cells in our adenoviral delivery system (Supplementary information, Figure S4B). For efficiency calculation, we adopted the efficiency calculation method 

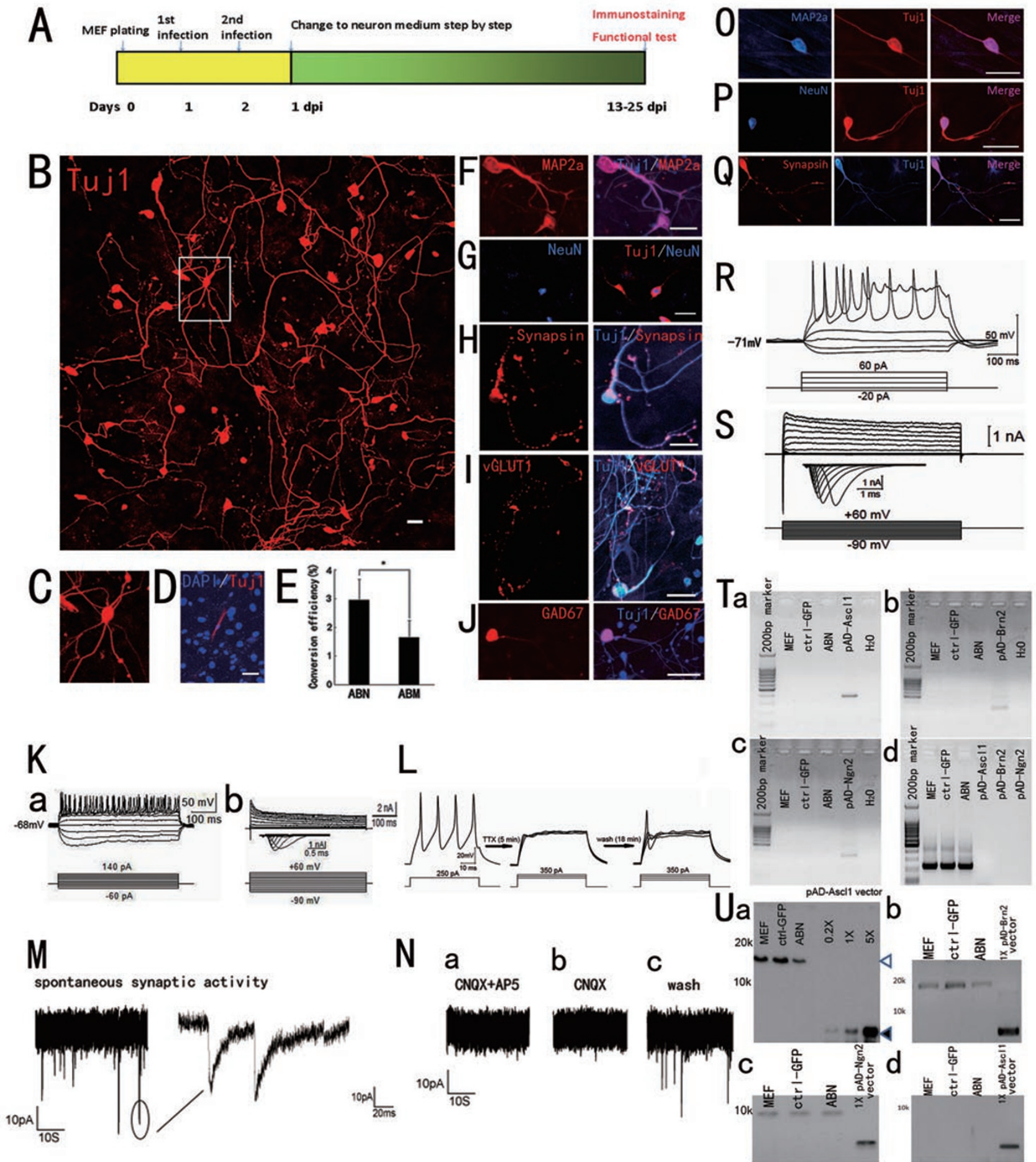

described by Vierbuchen et al. [1]. We randomly selected 8-10 visual fields for each well and calculated the total cell number visualized after DAPI staining and the total iN cell number indicated by Tuj1 staining. The efficiency was calculated by dividing the average number of iN cells by the number of total cells in each visual field. We repeated the experiment 4 times and at least 3 wells were analyzed for each experiment. Importantly, the ABN group showed a slightly higher efficiency $(2.9 \% \pm 0.7 \%)$ in generating iN cells than the ABM group $(1.6 \% \pm 0.6 \%)$ (Figure 1E). These ABN-iN cells also expressed the panneuronal markers MAP2a, NeuN and synapsin (Figure 
$1 \mathrm{~F}-1 \mathrm{H})$.

To validate that the fibroblasts were directly converted to iN cells rather than first transformed into transient progenitor intermediates, Nestin and Tuj1 double-immunostaining was conducted daily from the day before infection to $5 \mathrm{dpi}$. Tuj1-positive iN cells initially appeared at 3 dpi (Supplementary information, Figure S5A). At $5 \mathrm{dpi}$, increased numbers of Tuj1-positive iN cells were observed with typical neuronal morphology (Supplementary information, Figure S5B). During the process of transdifferentiation, however, Nestin-positive cells were not observed (data not shown). Thus, the infected MEFs were successfully converted to neurons using the nonintegrating adenovirus carrying a new combination of transcription factors: Ascl1, Brn2, and Ngn2.

To ascertain the functional membrane properties of adenovirus-induced neurons, we performed patch-clamp recordings of cells with neuronal morphology shown by fluorescence at 16-24 dpi (Supplementary information, Data S1). By step-depolarizing the membrane in currentclamp mode, action potentials (APs) were evoked in both ABN- (four out of six cells recorded) (Figure $1 \mathrm{Ka}$ ) and ABM-iN cells (four out of eight cells recorded) (Supplementary information, Figure S4C). In voltage-clamp mode, step-depolarization induced fast and inactivating inward sodium currents and outward potassium currents, as well as possible calcium currents (Figure $1 \mathrm{~Kb}$ and Supplementary information, Figure S4D and S4E). Therefore, we successfully generated functional iN cells from MEFs with both the reported combination of ABM and the new combination of $\mathrm{ABN}$ using our adenoviral system.

APs and inward sodium currents were abolished by a specific inhibitor of $\mathrm{Na}^{+}$ion channels, tetrodotoxin (TTX). The AP resumed following a washing period (Figure 1L and Supplementary information, Figure S6). APs and inward/outward currents of $\mathrm{ABN}-\mathrm{iN}$ cells were also recorded at 30 dpi (data not shown) and showed no significant difference between the two time points.

To explore the neurotransmitter phenotype of ABNiN cells, immunostaining was conducted on infected MEFs cultured for a longer period (20-25 dpi). Distinct vGLUT1-positive neurons (Figure 1I) $(28.4 \% \pm 4.4 \%$ of Tuj1-positive cells) and a much smaller population of GAD67-positive neurons $(1.3 \% \pm 0.9 \%)$ were detected (Figure 1J). To determine whether ABN-iN cells were capable of forming synapses, neonatal hippocampal neurons were isolated and incubated with iN cells at $10 \mathrm{dpi}$. After 10 days of co-culture with primary hippocampal neurons, ABN-iN cells frequently displayed spontaneous

Figure 1 (A) Diagram depicting the transdifferentiation procedures of MEFs into neurons by adenoviruses carrying either $A B N$ or ABM. (B) Tuj1 staining for MEFs infected with adenovirus carrying ABN at 13 dpi. (C) iN cells with highly complex neuronal morphologies are readily detected. (D) Control group infected with adenovirus carrying only eGFP showed few Tuj1-positive cells with fibroblast morphology. (E) Efficiencies from MEFs to neurons with adenoviral ABM and ABN $(n=6)$. The data are represented as \pm s.d. of cell counts. (F-H) At 13-15 dpi with adenovirus carrying ABN, MEF-derived iN cells coexpressed the pan-neuronal markers MAP2a (F), NeuN (G) and Synapsin (H) along with Tuj1. (I, J) After a longer culture period of 16-25 days, MEF-derived iN cells express a marker for excitatory neurons, vGLUT1 (I), as well as a marker for inhibitory neurons, GAD67 (J). (K) a Representative traces of action potentials in ABN iN cells evoked by step-depolarizing the membrane in current-clamp mode. b Representative traces of whole-cell currents in voltage-clamp mode of ABN iN cells. The lower panels show that iN cells were held at $-55 \mathrm{mV}$, and step depolarization at $10 \mathrm{mV}$ intervals was applied from $-90 \mathrm{mV}$ to $+60 \mathrm{mV}$. (L) Action potentials were abolished after the application of TTX. When TTX was washed away extracellularly, the action potentials resumed. (M) Spontaneous postsynaptic currents (PSCs) recorded from ABN iN cells co-cultured with hippocampal neurons. (N) a PSCs were largely abolished after the application of CNQX (blocker of the AMPA/Kainate receptor) and AP5 (blocker of the NMDA receptor). b PSCs were still barely detected after the application of CNQX. c With all blockers washed away from the extracellular solution, EPSCs resumed. (O-Q) ETF-derived iN cells co-expressed the pan-neuronal markers MAP2 (O), NeuN (P) and Synapsin (Q). (R) APs in response to step current injections of ETF-derived iN cells. (S) Whole-cell currents recorded by depolarizing voltage steps (-90 mV to $60 \mathrm{mV}$ ) in ETF-derived iN cells. (T) a-c PCR analysis for viral integration of genomic DNA extracted from uninfected MEFs, MEFs infected with ctrl-GFP virus, and MEFs infected with ABN. Plasmid containing cDNA of Ascl1, Brn2 or Ngn2 served as a positive control, respectively, and distilled water served as a negative control. d PCR analysis of GAPDH amplified from the genomic DNA of the three previously mentioned groups with the same dose of template. (U) Southern blot analysis of EcoRI-digested genomic DNA and plasmid DNA using DNA fragments amplified using primers (Supplementary information, Table S1) as probes: a Filled arrowheads indicating the position of EcoRI-digested fragments of adenoviral vector cross-hybridized to the Ascl1 probe; open arrowheads highlighting the position of EcoRI-digested fragments of genomic DNA cross-hybridized to the Ascl1 probe; plasmid DNA of pAD-Ascl1IRES-eGFP diluted to $0.2,1$, or 5 integrations per genome. b Detection of integration by the Brn2 probe, 1× plasmid DNA of pAD-Brn2-IRES-eGFP serving as the positive control. c Detection of integration by the Ngn2 probe, 1× plasmid DNA of pADNgn2-IRES-eGFP serving as the positive control. d Detection of integration by a probe randomly selected on the vector backbone, $1 \times$ plasmid DNA of pAD-Asc/1-IRES-eGFP serving as the positive control. Scale bars: $50 \mu \mathrm{m}$. 
synaptic activities (Figure 1M). In most of the iN cells recorded (three out of four), excitatory postsynaptic currents (EPSCs) were detected. In the presence of a blocker combination of CNQX (6-cyano-7-nitroquinoxaline2,3-dione, AMPA/Kainate receptor antagonist) and AP5 ((2R)-amino-5-phosphonovaleric acid, NMDA receptor antagonist), postsynaptic currents (PSCs) were largely blocked (Figure 1Na). Moreover, PSCs were largely abolished by the CNQX blocker alone (Figure $1 \mathrm{Nb}$ ), further indicating that the recorded PSCs were mainly AMPA-mediated glutamatergic synaptic currents. When CNQX was washed away from the extracellular solution, PSCs were detected again (Figure 1Nc).

To determine whether ABN-iN cells could also be derived from adult fibroblasts, ear-tip fibroblasts (ETFs) were isolated from 6-week-old C57/BL6 mice. ETFs were found to be fibronectin-positive and Tuj1/GFAP/ Nestin-negative (data not shown). At 15 dpi, Tuj1positive ETF-iN cells were observed, but with a lower efficiency than those derived from MEFs (data not shown). ETF-iN cells also expressed pan-neuronal markers MAP2, NeuN and Synapsin (Figure 1O-1Q) and demonstrated electrophysiological signals of APs, inward sodium currents and outward potassium currents (Figure $1 \mathrm{R}$ and $1 \mathrm{~S})$.

Different from retroviruses and lentiviruses, adenoviruses rarely integrate into the host genome [12]. To check for viral integration, PCR and Southern blot techniques were performed using genomic DNA (Supplementary information, Data S1). Genomic DNA was extracted from three groups: uninfected MEFs, MEFs infected with control virus (ctrl-eGFP), and MEFs infected with adenoviruses carrying ABN. Plasmid DNA of pAD-Ascll/ Brn2/Ngn2-IRES-eGFP was used as positive controls for the respective PCR reactions, while distilled water was used as a negative control. No predicted bands amplified by PCR were detected except in the positive control lane (Figure 1Ta-c). To ascertain the same quantities of PCR templates, the housekeeping gene GAPDH was simultaneously amplified with the same amount of genomic DNA from each of the three groups (Figure 1Td).

Southern blot was employed using the DNA fragments amplified by primers (Supplementary information, Table $\mathrm{S} 1)$ as probes to further exclude the possibility of viral integration. Genomic DNA from the three groups described above and plasmid DNA were digested by EcoRI. Only endogenous Ascl1/Brn2/Ngn2 was detected, and no bands of exogenous Ascl1/Brn2/Ngn2 were observed in the genomic DNAs from each group. Additionally, the DNA plasmids served as positive controls, indicating the existence and relative positions of the exogenous Ascll/ Brn2/Ngn2 (Figure 1Ua-c). To rule out genomic integra- tion of the remaining sequences of the adenoviral vector, we randomly selected a sequence on the vector (detected by primer pAD-random-F, and pAD-random-R; Supplementary information, Table S1) and did not detect this exogenous viral sequence in the genomic DNA from the three groups (Figure 1Ud).

In our study, we successfully converted mouse embryonic and adult fibroblasts to functional neurons with the adenoviral delivery system for the first time. We chose a novel transcription factor combination of $\mathrm{ABN}$ rather than the previously reported ABM. We replaced the reported Myt1l [1] with Ngn2 because Ngn2 alone could reprogram astroglia to functional neurons with an efficiency of $85.4 \%$, as indicated by MAP2 immunostaining [10]. However, Ngn2 alone could not induce neurons from fibroblasts in our study (data not shown).

By comparing the conversion efficiencies estimated by Tuj1-positive cells between the two groups, we found that the ABN group had a slightly higher efficiency $(2.9 \%$ $\pm 0.7 \%)$ than the ABM group $(1.6 \% \pm 0.6 \%)$. However, the conversion efficiency in our adenoviral system still seemed much lower than that in the integrating lentiviral system (approximately $2.9 \%$ versus $19.5 \%$ [1]). An explanation for this discrepancy may be the transient expression of exogenous genes carried by adenoviruses rather than the persistent expression of those carried by lentiviruses. This efficiency difference coincided with the results of iPSC generation: lentiviruses displayed a 10 fold higher efficiency than adenoviruses $(0.001 \%-0.03 \%$ versus $0.0001 \%-0.001 \%)[13,14]$.

ABN-iN cells expressed the neuronal markers of Tuj1, Map2a, Synapsin and NeuN, exhibited APs, and developed functional synapses when co-cultured with primary hippocampal neurons. Typical APs could be elicited in the majority of MEF-derived iN cells at 17-25 dpi. However, the ETF-derived iN cells (Figure 1R) could only sustain low current input in contrast to MEF-iN cells (Figure 1Ka), and fewer APs were stimulated (Figure 1R). No more APs could be elicited by further membrane depolarization. Some of the ETF-iN cells with ideal morphology could only exhibit a single AP, which was rarely observed in MEF-derived iN cells. A longer period of culture did not seem to change much on the membrane properties of ETF-derived iN cells (data not shown). It is possible that it was relatively difficult to convert adult fibroblasts (from 6-week-old mouse) to mature neurons. Our further studies will focus on how to improve the direct reprogramming of adult somatic cells to functional neurons, both in vitro and in vivo, to explore the clinical applications of the adenoviral delivery system.

Although the generation of iN cells skipped the generation of iPSCs, which could be tumorigenic, lentiviral 
delivery technology hindered clinical applications, especially in vivo therapies. In our study, the transgenes did not integrate into the genome of infected fibroblasts as shown by PCR and Southern blot analysis. Intriguingly, the fibroblasts that were not destined to become neurons gradually lost GFP expression within about 15 days, while iN cells could sustain it over 30 days. It is possible that those fibroblasts would keep dividing when they were not infected with the proper dose of adenoviruses. Thus, the GFP expression level diluted quickly as the cells divided. Those cells that received the proper dose of transgenes would stop dividing and initiate their transition to neurons. It is well known that neurons seldom divide, which might explain why the GFP expression level is maintained for a certain period of time until the protein is degraded. Therefore, iN cells were readily detected by fluorescence until 30 dpi. GFP served as an excellent indicator for us to trace the changes of iN cells and facilitated the electrophysiological conduction as well.

In this study, we directly converted mouse fibroblasts to neurons with a non-integrating adenoviral delivery system for the first time. We chose a different combination of three transcription factors to obtain higher conversion efficiency, although the efficiency was much lower than that of the lentiviral delivery system. In the future, we will focus on how to increase the efficiency of our system, either by exploring other genes important for iN cell generation or by optimizing the conditions of infection and culturing. We will also attempt to generate human iN cells with the adenoviral delivery system to provide an encouraging prospect for the application of iN cells in regenerative medicine.

\section{Acknowledgments}

This work was supported by grants from the Ministry of Science and Technology of China (2011CBA00400), the Chinese Academy of Sciences (Hundred Talent Program) and the Stem Cell Program (XDA01020301, XDA01020305).

Fengxi Meng ${ }^{1, *}$, Siye Chen ${ }^{2, *}$, Qinglong $\mathrm{Miao}^{2}$, Kechun Zhou', Qicheng Lao ${ }^{3}$, Xiaohui Zhang', Wenyi Guo ${ }^{1,4}$, Jianwei Jiao ${ }^{5}$

${ }^{l}$ Department of ophthalmology, Eye \& ENT Hospital, Fudan University, 83 Fenyang Rd, Shanghai 200031, China; ${ }^{2}$ Institute of Neuroscience, State Key Laboratory of Neuroscience, Shanghai Institutes for Biological Sciences, Chinese Academy of Sciences, 320 Yueyang Rd, Shanghai 200031, China; ${ }^{3} \mathrm{Fu}$ dan University Shanghai Medical College, Shanghai 200032, China; ${ }^{4}$ Department of Ophthalmology, Ninth People's Hospital, Shanghai Jiao Tong University School of Medicine, 639
Zhizaoju Rd, Shanghai 200011, China; ' Institute of Zoology, State Key Laboratory of Reproductive Biology, Chinese Academy of Sciences, 1 Beichen West Rd, Beijing 100101, China

*These two authors contributed equally to this work.

Correspondence: Jianwei Jiao ${ }^{\mathrm{a}}$, Wenyi Guo ${ }^{\mathrm{b}}$

${ }^{\mathrm{a}}$ Tel: +86-10-64806229; Fax: +86-10-64806229

E-mail: jwjiao@ioz.ac.cn

${ }^{\mathrm{b}}$ Tel: + 86-21-23271699; Fax: + 86-21-63136856

E-mail:wyguo@163.com

\section{References}

1 Vierbuchen T, Ostermeier A, Pang ZP, Kokubu Y, Südhof TC, Wernig M. Direct conversion of fibroblasts to functional neurons by defined factors. Nature 2010; 463:1035-1041.

2 Wernig M, Zhao JP, Pruszak J, et al. Neurons derived from reprogrammed fibroblasts functionally integrate into the fetal brain and improve symptoms of rats with Parkinson's disease. Proc Natl Acad Sci USA 2008; 105:5856-5861.

3 Huang P, He Z, Ji S, et al. Induction of functional hepatocytelike cells from mouse fibroblasts by defined factors. Nature 2011; 475:386-389.

4 Pang ZP, Yang N, Vierbuchen T, et al. Induction of human neuronal cells by defined transcription factors. Nature 2011; 476:220-223.

5 Pfisterer U, Kirkeby A, Torper O, et al. Direct conversion of human fibroblasts to dopaminergic neurons. Proc Natl Acad Sci USA 2011; 108:10343-10348.

6 Caiazzo M, Dell'Anno MT, Dvoretskova E, et al. Direct generation of functional dopaminergic neurons from mouse and human fibroblasts. Nature 2011; 476:224-227.

7 Kim J, Efe JA, Zhu S, et al. Direct reprogramming of mouse fibroblasts to neural progenitors. Proc Natl Acad Sci USA 2011; 108:7838-7843.

8 Ieda M, Fu JD, Delgado-Olguin P, et al. Direct reprogramming of fibroblasts into functional cardiomyocytes by defined factors. Cell 2010; 142:375-386.

9 Szabo E, Rampalli S, Risueno RM, et al. Direct conversion of human fibroblasts to multilineage blood progenitors. Nature 2010; 468:521-526.

10 Heinrich C, Blum R, Gascon S, et al. Directing astroglia from the cerebral cortex into subtype specific functional neurons. PLoS Biol 2010; 8:e1000373.

11 Berninger B, Costa MR, Koch U, et al. Functional properties of neurons derived from in vitro reprogrammed postnatal astroglia. J Neurosci 2007; 27:8654-8564.

12 Harui A, Suzuki S, Kochanek S, Mitani K. Frequency and stability of chromosomal integration of adenovirus vectors. $J$ Virol 1999; 73:6141-6146.

13 Stadtfeld M, Nagaya M, Utikal J, Weir G, Hochedlinger K. Induced pluripotent stem cells generated without viral integration. Science 2008; 322:945-949.

14 Okita K, Ichisaka T, Yamanaka S. Generation of germlinecompetent induced pluripotent stem cells. Nature 2007; 448:313-317.

(Supplementary information is linked to the online version of the paper on the Cell Research website.) 